\title{
The Sun as a fundamental calibrator of stellar evolution
}

\author{
Jørgen Christensen-Dalsgaard \\ Danish Asteroseismology Centre, and Department of Physics and Astronomy, \\ Aarhus University, DK 8000 Aarhus C, Denmark \\ email: jcd@phys.au.dk
}

\begin{abstract}
The Sun is unique amongst stars in having a precisely determined age which does not depend on the modelling of stellar evolution. Furthermore, other global properties of the Sun are known to much higher accuracy than for any other star. Also, helioseismology has provided detailed determination of the solar internal structure and rotation. As a result, the Sun plays a central role in the development and test of stellar modelling. Here I discuss solar modelling and its application to tests of asteroseismic techniques for stellar age determination.
\end{abstract}

Keywords. Sun: evolution, Sun: interior, Sun: helioseismology, Sun: fundamental parameters, stars: evolution, stars: interior, stars: oscillations, stars: fundamental parameters, asteroseismology

\section{Introduction}

Determining the age of a star from its observed properties requires a model describing how those properties change as the star evolves. Amongst the many properties discussed in this volume, those relevant to using the Sun as a calibrator depend on the changes in the internal structure of the star caused by evolution. Specifically, the change in the composition as hydrogen is fused to helium (with the additional effects of diffusion and settling) changes the internal structure and hence the observable properties. Evidently, both the details of the composition change, and the response of the structure and the observables, depend on the modelling of stellar interiors, and hence the age determination is sensitive to uncertainties in the modelling. The Sun provides a unique possibility for quantifying these uncertainties and attempting to reduce them.

In the present case the Sun has several major advantages: it is the only star for which the age can be inferred in a manner that is essentially independent of stellar modelling, through the radioactive dating of the solar system. Also, its proximity means that its mass, radius and luminosity are known to high accuracy. Finally, as a result of this proximity, helioseismology based on a broad range of modes has allowed inferences to be made of the detailed internal structure (and rotation) of the Sun. This allows a refined test of the modelling of stellar evolution. In addition, by applying techniques for age determination, based on stellar modelling, to the Sun we can test and possibly improve them. This, in particular, applies to the use of asteroseismic analyses for age determination; as discussed by Lebreton \& Montalbán (this volume) these promise to be far more precise than other techniques based on the evolution of stellar structure.

\section{Solar modelling}

A summary of solar modelling and the helioseismic investigations of the Sun was given, for example, by Christensen-Dalsgaard (2002). Bahcall et al. (2006) made a detailed 
analysis of the sensitivity of the models to the choice of input parameters and physics. The solar models used here largely correspond to the so-called Model S of ChristensenDalsgaard et al. (1996), although with some updates.

The mass of the Sun is known from planetary motion, with an accuracy limited by the accuracy of the determination of the gravitational constant $G$. For the modelling presented here I use $G=6.67232 \times 10^{-8}$ in $\mathrm{cgs}$ units, and hence the solar mass is $M_{\odot}=1.989 \times 10^{33} \mathrm{~g}$. The solar radius $R_{\odot}$ is obtained from the solar angular diameter and the distance to the Sun; the radius should be defined in a manner that can be related precisely to the model, e.g., as the distance from the centre to the photosphere, defined by the location where the temperature is equal to the effective temperature. A commonly used value is $R_{\odot}=6.9599 \times 10^{10} \mathrm{~cm}$ (Auwers 1891), and this value is used here. However, I note that Brown \& Christensen-Dalsgaard (1998) obtained the value $6.9551 \times 10^{10} \mathrm{~cm}$ which is probably more accurate. The solar luminosity $L_{\odot}$ is obtained from the solar constant, i.e., solar flux at the Earth, and the distance to the Sun; this assumes that the solar flux does not depend on latitude, a probably reasonable assumption although one that has never been verified; I use the value $L_{\odot}=3.846 \times 10^{33} \mathrm{erg} \mathrm{s}^{-1}$ (see also Fröhlich \& Lean 2004).

The solar photospheric composition can be determined from spectral analysis. An important exception is the abundance of helium: the helium lines that led to the detection of helium in the Sun (and hence the name of the element) are formed in the chromosphere, under conditions such that the abundance determination is quite inaccurate. The abundances of other noble gases, amongst which neon is a relatively important constituent of the solar atmosphere, are similarly inaccurate. Thus only relative abundances, commonly defined relative to hydrogen, can be determined. In solar modelling this is typically characterized by the ratio $Z_{\mathrm{s}} / X_{\mathrm{s}}$ of the surface abundances by mass $Z_{\mathrm{s}}$ of elements heavier than helium and $X_{\mathrm{s}}$ of hydrogen. A commonly used value has been $Z_{\mathrm{s}} / X_{\mathrm{s}}=0.0245$ (Grevesse \& Noels 1993). However, redeterminations of the abundances, based on threedimensional hydrodynamical models of the solar atmosphere and taking departures from local thermodynamical equilibrium into account, have had a major impact on the inferred abundances; in particular, the abundances of oxygen, carbon and nitrogen were substantially reduced (e.g., Asplund et al. 2004) (for a review, see also Asplund 2005). This resulted in $Z_{\mathrm{s}} / X_{\mathrm{s}}=0.0165$; as discussed below, this has had drastic consequences for the comparison between solar models and the helioseismically inferred structure.

For the present discussion the solar age is of course of central importance. This can be inferred from radioactive dating of material from the early solar system, as represented by suitable meteorites. In a detailed discussion presented in the appendix to Bahcall \& Pinsonneault (1995), G. J. Wasserburg concluded that the age of the Sun, since the beginning of its main-sequence evolution, is between $4.563 \times 10^{9}$ and $4.576 \times 10^{9} \mathrm{yr}$. The rounded value of $4.6 \times 10^{9} \mathrm{yr}$ is often used, including for the reference Model S.

It is evident that computed solar models should match the observed quantities, at the age of the Sun. Models are typically computed without mass loss (see, however, Sackmann \& Boothroyd 2003) and hence with the present mass of the Sun. A model with the correct radius, luminosity and $Z_{\mathrm{s}} / X_{\mathrm{S}}$ is obtained by adjusting three parameters that are a priori unknown: a parameter, such as the mixing-length parameter $\alpha_{\mathrm{ML}}$, $\dagger$ characterizing the properties of convection which largely determines the radius, the initial helium abundance $Y_{0}$ which mainly determines the luminosity, and the initial heavy-element abundance $Z_{0}$ which determines $Z_{\mathrm{s}} / X_{\mathrm{s}}$. This calibration provides a precise (although not necessarily

$\dagger$ i.e., the ratio of the mixing length to the pressure scale height in the mixing-length description of convection (e.g., Böhm-Vitense 1958; Gough \& Weiss 1976) 
accurate) determination of the initial solar helium abundance, of importance to studies of galactic chemical evolution. Also, the resulting value of $\alpha_{\mathrm{ML}}$ is often used for computations of other stellar models, although there is little justification for regarding $\alpha_{\mathrm{ML}}$ as being independent of stellar parameters. More significantly, the calibration of the properties of the solar convection zone can be used as a test of hydrodynamical simulations of convection (e.g., Demarque et al. 1999; Rosenthal et al. 1999), these may then be used to calibrate the dependence of convection-zone properties, e.g., characterized by $\alpha_{\mathrm{LM}}$, on stellar parameters (Ludwig et al. 1997, 1999; Trampedach et al. 1999).

An important solar observable is the neutrino flux. The discrepancy between the predicted and detected flux of electron neutrinos was long regarded as a potential problem of solar modelling, although even early helioseismic results strongly indicated that changes to the models designed to eliminate the discrepancy were inconsistent with the observed oscillation frequencies (e.g., Elsworth et al. 1990). However, it is now realized that the apparent discrepancy was caused by oscillations between different states of the neutrino; with the recent detection of neutrinos of other flavours the total observed flux of neutrinos agrees with predictions (Ahmad et al. 2002). Thus the emphasis in the study of solar neutrinos has shifted towards the investigation of the detailed properties of the neutrino oscillations (for recent reviews, see Bahcall et al. 2004; Robertson 2006; Haxton et al. 2006; Haxton 2008). Interestingly, the computed neutrino flux is not significantly affected by the recent revision in the solar composition (e.g. Bahcall \& Serenelli 2005).

\section{Helioseismic results on the solar interior}

Very extensive data on solar oscillations have been obtained in last two decades (for a review, see Christensen-Dalsgaard 2002). Unlike any other pulsating star the availability of observations with high spatial resolution has provided accurate frequencies for modes over a broad range of spherical-harmonic degrees $l$, from 0 to more than 1000 (see also Lebreton \& Montalbán, these proceedings, for an overview of the properties of stellar oscillations). Most of the observed modes are acoustic modes; these are essentially trapped between the solar surface and an inner turning point at a distance $r_{\mathrm{t}}$ from the centre given by $c\left(r_{\mathrm{t}}\right) / r_{\mathrm{t}}=\omega / \sqrt{l(l+1)}$, where $c$ is the adiabatic sound speed and $\omega$ is the angular frequency of the mode. Thus the broad range of $l$ corresponds to a range of inner turning points varying from the centre to just beneath the solar surface. This availability of modes sensitive to very different parts of the Sun is essentially what allows inverse analyses to resolve the structure and rotation of the solar interior.

In the analysis of solar and solar-like pulsations an important issue is the effect of the near-surface layers: modelling of the structure of these layers and of their effect on the oscillation frequencies is highly uncertain, leading to systematic errors in the computed frequencies, which in many cases dominate the differences between observed and computed solar frequencies (e.g., Christensen-Dalsgaard et al. 1988). These errors depend essentially only on frequency (apart from a trivial dependence on the mode inertia) and furthermore are small at low frequency (e.g., Christensen-Dalsgaard \& Thompson 1997). This allows their effect to be eliminated in the analysis of solar data but they should be kept in mind also in asteroseismic analyses (Kjeldsen et al. 2008a).

To illustrate the inference of solar structure, Fig. 1 shows the inferred difference in squared sound speed between the Sun and two solar models. One is what might be termed a 'standard' model, computed with the Grevesse \& Noels (1993) composition. Here the relative differences are below $0.5 \%$; although this is far more than the very small estimated errors in the difference it still indicates that the model provides a good representation of the solar interior. Also shown are the results for a corresponding model computed 
with the revised (Asplund 2005) composition. It is evident that this leads to a dramatic deterioration in the agreement between the model and the Sun (see also Basu \& Antia 2008, for a review). Similar discrepancies are found for other helioseismically inferred quantities, such as the depth of the convection zone and the envelope helium abundance. Of particular relevance to the present discussion is the inconsistency found in a detailed analysis by Chaplin et al. (2007) in the small frequency separation $\delta \nu_{l l+2}(n)=\nu_{n l}-$ $\nu_{n-1} l+2, \nu_{n l}$ being the cyclic frequency of a mode of degree $l$ and radial order $n$; as discussed by Lebreton \& Montalbán (this volume) this is a measure of stellar age. I return to the consequences of this below.

These discrepancies clearly indicate potential problems with solar modelling, if the revision of the solar abundances is accepted. Guzik $(2006,2008)$ reviewed the attempts to modify the model calculation to restore the agreement with helioseismology which so far have not led to any entirely satisfactory solution. A trivial modification is to postulate intrinsic errors in the opacity tables which compensate for the composition change (Bahcall et al. 2005; Christensen-Dalsgaard et al. 2009); however, as discussed in the latter reference the required change is as high as $30 \%$ at the base of the convection zone, at a temperature of $2 \times 10^{6} \mathrm{~K}$, which may be unrealistic. A resolution of these issues is evidently of general importance to stellar modelling and hence to the determination of stellar ages from evolution calculations.

I finally recall that helioseismology has yielded detailed inferences of the solar internal rotation (see Thompson et al. 2003, for a review). This shows that the convection zone approximately shares the surface latitudinal differential rotation, while the radiative interior rotates at a nearly constant rate, somewhat smaller than the surface equatorial rotation rate. This is obviously relevant to the modelling of the, so far incompletely understood, evolution of stellar rotation and hence to the use of gyrochronology for stellar age determinations (Barnes 2007, Meibom, this volume).

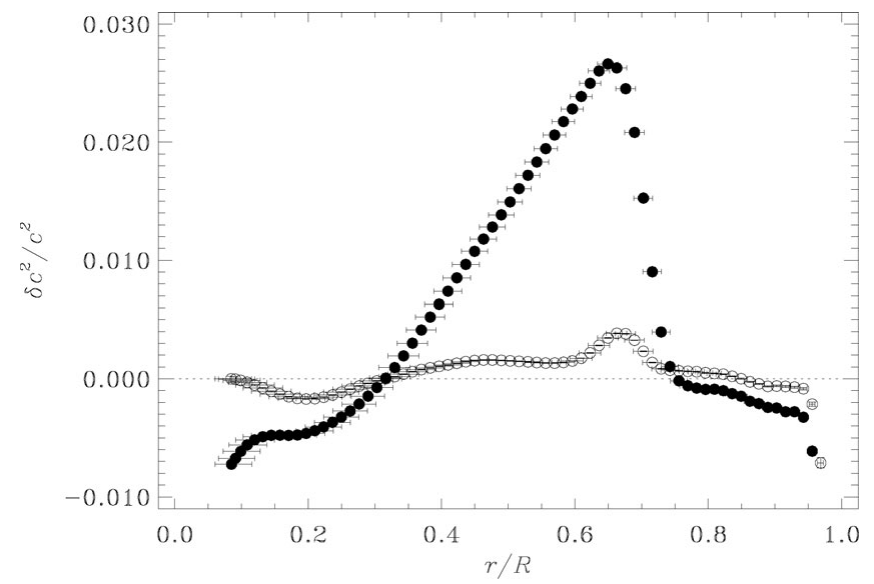

Figure 1. Inferred relative differences in squared sound speed, in the sense (Sun) - (model), from inversion of frequency differences between the Sun and two solar models. The open circles used Model S of Christensen-Dalsgaard et al. (1996), based on the Grevesse \& Noels (1993) composition, while the filled circles are for a corresponding model but using the revised solar composition (Asplund 2005). The horizontal bars provide a measure of the resolution of the inversion. The standard error in the inferred differences are generally smaller than the size of the symbols. (From Christensen-Dalsgaard et al. 2009). 


\section{Asteroseismic age determination}

As discussed by Lebreton \& Montalbán (this volume), asteroseismology† provides sensitive diagnostics of stellar ages. The change in the internal structure of a star with evolution directly affects the oscillation frequencies, and hence the observed frequencies, when suitably analyzed, can be used to determine the age. Also, the computation of the relevant aspects of the frequencies from a given model structure is relatively insensitive to systematic errors; on the other hand, the dependence of the structure on age is clearly affected by uncertainties in the modelling.

Here I concentrate on acoustic modes in solar-like stars; these are typically of high radial order and hence their diagnostic potential can be investigated on the basis of asymptotic theory. The low-degree modes that are relevant to observations of distant stars penetrate to the core of the star; hence their frequencies are sensitive to the sound speed in the core and consequently to the composition change resulting from evolution. In the ideal-gas approximation $c^{2} \propto T / \mu$, where $T$ is temperature and $\mu$ is the mean molecular weight. Both increase as a result of evolution; however, since the temperature is strongly constrained by the high temperature sensitivity of the nuclear burning rates, the change in $\mu$ dominates, leading to a decrease in the sound speed in the core. This is illustrated in Fig. 2 for the evolution of a $1 M_{\odot}$ star. The decrease in $c$ affects most strongly the modes of the lowest degree which penetrate most deeply; consequently, the small frequency separations $\delta \nu_{02}$ and $\delta \nu_{13}$ decrease with increasing age. To characterize a star based on high-order acoustic-mode frequencies one can in addition use the large frequency separation $\Delta \nu_{n l}=\nu_{n l}-\nu_{n-1 l}$ which essentially provides a measure of the mean density of the star. Thus the position of the star in a $(\langle\Delta \nu\rangle,\langle\delta \nu\rangle)$ diagram, based on suitable averages, provides an indication of the mass and age of the star (ChristensenDalsgaard 1984, 1988; Ulrich 1986). Obviously, the calibration of the diagram depends on the physics and other parameters, such as the composition, of the stars (Gough 1987; Monteiro et al. 2002, see also Lebreton \& Montalbán, these proceedings). These potential systematic errors must be taken into account in the interpretation of the results.

$\dagger$ For an illuminating and entertaining discussion of the etymology of asteroseismology, see Gough (1996).
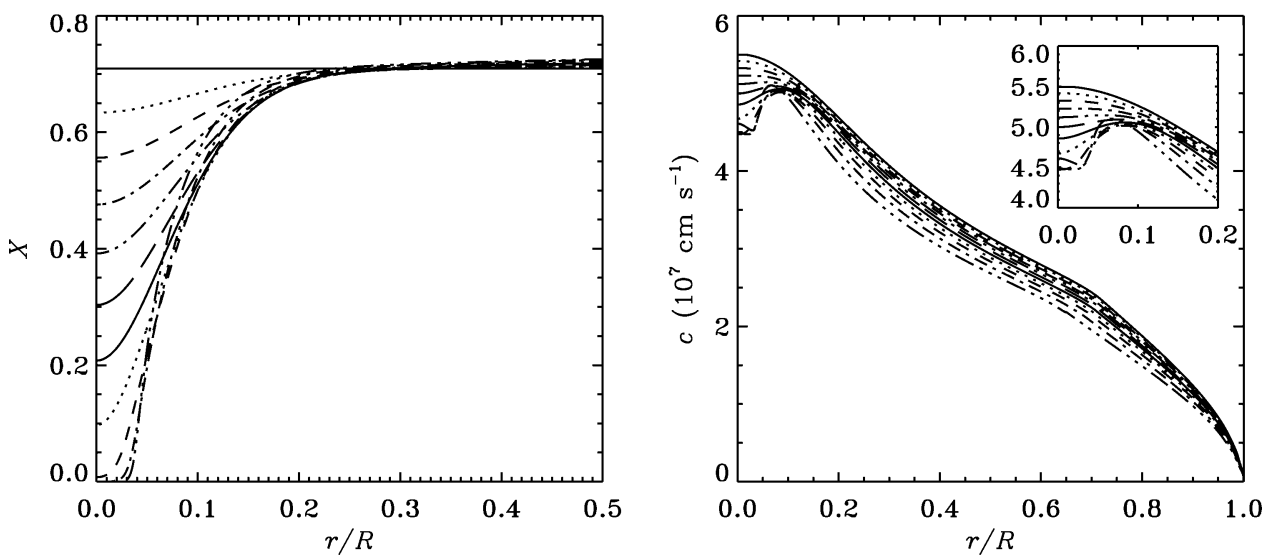

Figure 2. The left-hand panel shows the hydrogen abundance $X$ in the inner part of $1 M_{\odot}$ models of age $0-10 \mathrm{Gyr}$, in steps of $1 \mathrm{Gyr}$. The right-hand panel shows the sound speed in these models, with an enlargement in the insert of the behaviour in the core. Except for the final model the central sound speed decreases with increasing age. 
It was noted by Roxburgh \& Vorontsov (2003) that the near-surface errors, although to a large extent canceling in the difference, has a significant effect on the small frequency separations. They showed that this effect is suppressed by evaluating separation ratios, such as

$$
r_{02}(n)=\frac{\nu_{n 0}-\nu_{n-12}}{\nu_{n 1}-\nu_{n-11}}, \quad r_{13}(n)=\frac{\nu_{n 1}-\nu_{n-13}}{\nu_{n+10}-\nu_{n 0}},
$$

and demonstrated that these ratios are directly related to the effect of the stellar core on the oscillation frequencies. This was further analyzed by Otí Floranes et al. (2005) who showed that, unlike $\delta \nu_{02}, r_{02}$ is essentially insensitive to structure changes near the surface; they furthermore considered several examples of model modifications, including changes to the stellar radius, keeping the structure of the core unchanged and found that these did not affect the separation ratios. The use of $\left(\langle\Delta \nu\rangle,\left\langle r_{02}\right\rangle\right)$ diagrams to characterize stellar properties is discussed by Lebreton \& Montalbán (this volume).

This correction for the near-surface effects assumes that they are independent of degree and hence essentially that the underlying physical cause is spherically symmetric. In fact, it is known from the case of the Sun that the magnetic activity causes frequency changes that are strongly related to the distribution in latitude of the magnetic field (e.g., Howe et al. 2002). As noted by Dziembowski \& Goode (1997) the concentration of magnetic activity towards the equator causes a frequency shift for low-degree modes, observed with limited frequency resolution, that depends on degree and hence might corrupt the study of the solar core based, e.g., on the small frequency separations. Such degreedependent frequency shifts were in fact observed by Chaplin et al. (2004) and Toutain \& Kosovichev (2005). It was argued by Dziembowski \& Goode (1997) that the effects could be eliminated in the solar case from observations of higher-degree modes; however, it is obvious that they are a significant concern in observations of distant stars where only low-degree data are available.

The independent radioactive age determination of the solar system provides an excellent test of the use of the oscillation frequencies of low-degree acoustic modes to determine stellar ages. Gough \& Novotny (1990) made a careful analysis of the sensitivity of seismic age determinations to other aspects of the solar models. This was extended by Gough (2001), including also a determination of $Z_{\mathrm{s}} / X_{\mathrm{s}}$, from the analysis of $\delta \nu_{02}$ and $\delta \nu_{03}$; the results were consistent with the meteoritic ages although with a substantial uncertainty, owing to the strong sensitivity of the small frequency spacings to $Z_{\mathrm{s}} / X_{\mathrm{s}}$. Guenther \& Demarque (1997) also estimated the solar age based on the small frequency separations. More systematic analyses, using a $\chi^{2}$ fit to the observed values, fixing the value of $Z_{\mathrm{s}} / X_{\mathrm{s}}$, were carried out by Dziembowski et al. (1999) and Bonanno et al. (2002). These analyses showed that the seismically inferred age was in good agreement with the meteoritic age. Dziembowski et al. also found that the inferred age decreased with an increased $Z_{\mathrm{s}} / X_{\mathrm{s}}$, in accordance with the results of Gough (2001).

I have repeated this type of analysis, using the observed frequencies of Chaplin et al. (2007), based on 4572 days of observation with the BiSON network and corrected for frequency shifts caused by the solar magnetic activity. The models essentially corresponded to Model S of Christensen-Dalsgaard et al. (1996), except that more recent OPAL opacities (Iglesias \& Rogers 1996) and OPAL equation of state (Rogers \& Nayfonov 2002) $\dagger$

$\dagger$ In particular, the equation of state tables included relativistic effects for the electrons; Bonanno et al. (2002) found that these had a noticeable effect on the age fit. 
were used. The goodness of fit was determined by, for example,

$$
\chi^{2}\left(\delta \nu_{02}\right)=\frac{1}{N-1} \sum_{n} \frac{\left[\delta \nu_{02}(n)^{(\mathrm{obs})}-\delta \nu_{02}(n)^{(\mathrm{mod})}\right]^{2}}{\sigma\left[\delta \nu_{02}(n)\right]^{2}},
$$

where $N$ is the number of modes included, $\delta \nu_{02}(n)^{(\mathrm{obs})}$ and $\delta \nu_{02}(n)^{(\bmod )}$ are the observed and model values of the small separation, and $\sigma\left[\delta \nu_{02}(n)\right]^{2}$ is the variance of the observed small separation.

Preliminary results of this analysis are shown in Fig. 3. Fits have been made to both the unscaled small separations $\delta \nu_{02}$ and $\delta \nu_{13}$ and the separation ratios $r_{02}$ and $r_{13}$. I have computed results as functions of age, in all cases calibrating the models to the solar radius and luminosity and a fixed value of $Z_{\mathrm{s}} / X_{\mathrm{s}}$. The left-hand curves assumed $Z_{\mathrm{S}} / X_{\mathrm{S}}=0.0245$ (Grevesse \& Noels 1993); here the best fits are evidently obtained close to the age interval obtained from the meteoritic analysis, indicated by the vertical lines. Interestingly, the fit for the separation ratios indicate a slightly lower age and, in the case of $r_{13}$, a substantially lower $\chi^{2}$; in general, the values of $\chi^{2}$ show that the models are not entirely consistent with the observations. On the other hand, the results in the right-hand curves for the Asplund (2005) composition, with $Z_{\mathrm{s}} / X_{\mathrm{s}}=0.0165$, are clearly entirely inconsistent with the meteoritic age. This is in agreement with the analysis by Chaplin et al. (2007) of the small separations, similarly showing that they are incompatible with the revised composition. Also, the values of the minimal $\chi^{2}$ are much larger than for the GN93 composition. The very large minimum $\chi^{2}$ for $r_{02}$ in this case clearly requires further investigation. Note that the shift in the inferred age with $Z_{\mathrm{s}} / X_{\mathrm{s}}$ is in accordance with the results obtained by Dziembowski et al. (1999) and Gough (2001).

As noted by Gough (2002) the systematic errors, arising from the other unknown parameters of the stars, far exceed the effects of the statistical errors in the oscillation frequencies. To constrain these parameters on the basis of asteroseismic data he proposed
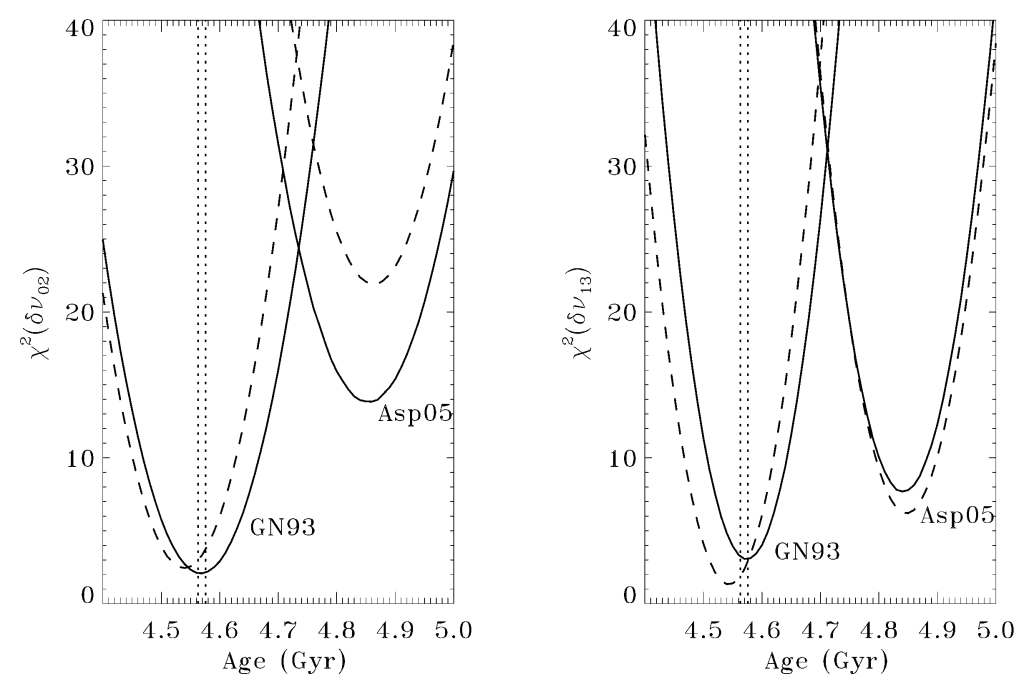

Figure 3. Fits of solar models, as a function of age, to the frequency separations obtained from BiSON observations (Chaplin et al. 2007); the left-hand panel shows results for the separations between $l=0$ and 2 , and the right-hand panel for separations between $l=1$ and 3 . The solid curves are for $\delta \nu_{l l+2}$ and the dashed curves for $r_{l l+2}$. Results are shown both for models computed with the Grevesse \& Noels (1993) composition (GN93) and the Asplund (2005) composition (Asp05). The vertical dotted lines indicate the interval of solar age obtained by Wasserburg, in Bahcall \& Pinsonneault (1995). 
the analysis of other aspects of the frequencies, particularly the effects sharp features in the sound speed (see also Lebreton \& Montalbán, this volume). Houdek \& Gough (2007a) made a detailed analysis of the effects of such features, which they called 'acoustical glitches', in order to derive reliable diagnostics for the envelope helium abundance and the depth of the convection zone. This was applied by Houdek \& Gough (2007b) and Houdek \& Gough (2008) to the determination of the solar age, based solely on low-degree frequencies such as might be observed in other stars; they noted that removing the effects of the glitches from the frequencies resulted in a more robust calibration for the age. The resulting age, calibrating the radius and luminosity to solar values but determining the heavy-element abundance from the fit, is close to, but not entirely consistent with, the meteoritic age; preliminary results are an age $t_{\odot}=(4.68 \pm 0.02) \mathrm{Gyr}$ and an initial heavy-element abundance $Z_{0}=0.0169 \pm 0.0005$.

It is evident that processes modifying the core composition have a potentially serious effect on the age determination. This is particularly true for stars with convective cores where the uncertain extent of convective overshoot has a large effect on the relation between age and stellar structure, as illustrated by Lebreton \& Montalbán (this volume) in a $\left(\langle\Delta \nu\rangle,\left\langle r_{02}\right\rangle\right)$ diagram. To detect such effects and correct the age determinations for them require asteroseismic analysis beyond the simple fits to the frequency separations. It is encouraging that it appears to be possible, with sufficiently good data, to resolve the structure of stellar cores in inverse analyses using just low-degree modes (e.g., Gough \& Kosovichev 1993; Basu et al. 2002; Roxburgh 2002). This may allow determination of the extent of convective overshoot and other mixing processes that could affect the age determination. Also, based on asymptotic analysis Cunha \& Metcalfe (2007) developed a diagnostic tool which may be used to characterize small convective cores and hence potentially eliminate the effects of additional mixing. A similar diagnostic was found by Mazumdar et al. (2006) on the basis of extensive model calculations.

\section{Next steps}

We have yet to see the full realization of the potential for age determination based on asteroseismology, but the observational prospects are excellent. The CoRoT mission has yielded the first results on solar-like stars (e.g., Appourchaux et al. 2008) and much more is expected in the next few years. The NASA Kepler mission, with planned launch in March 2009, will yield excellent asteroseismic data for a very large number of stars (e.g., Christensen-Dalsgaard et al. 2007; Kjeldsen et al. 2008b); an important aspect of the asteroseismic investigation based on Kepler data, given the main goal of the mission of characterizing extra-solar planetary systems, is to determine properties of the central stars in such systems, in particular their radius and age. In the longer term, groundbased projects for Doppler-velocity observations of stellar oscillations, such as the SONG (Grundahl et al. 2008) and SIAMOIS (Mosser et al. 2008) projects, are expected to yield exquisite data although for a smaller number of stars.

To utilize fully the data from these projects we need further development and tests of the asteroseismic diagnostic tools, taking into account also the additional unknown properties of the stars on the one hand, and other observed properties of the stars on the other. There is no doubt that, as in the past, asymptotic analyses will be extremely important guides in determining the optimal combinations of frequencies; however, extensive model calculations and analysis of artificial data, under the various relevant assumptions, will also be crucial. This clearly needs to take into account also the detailed properties of the oscillations and their effect on the inferred oscillation parameters (e.g., Chaplin et al. 2008a). A central effort in this regard is the asteroFLAG project (Chaplin et al. 2008b,c) 
to carry out blind tests on the analysis of artificial data, involving a substantial number of different techniques and groups.

As a result of these efforts, both observational and theoretical, we may hope to obtain reliable and precise age determinations for a number of stars of varying properties. These can then be used as calibrators for other, less direct, age diagnostics and thus extend the base for the general determination of stellar ages.

\section{References}

Ahmad, Q. R., Allen, R. C., Andersen, T. C., et al. 2002, Phys. Rev. Lett. 89, 011301

Appourchaux, T., Michel, E., Auvergne, M., et al. 2008, A\&A 488, 705

Asplund, M. 2005, ARAA 43, 481

Asplund, M., Grevesse, N., Sauval, A. J., Allende Prieto, C., \& Kiselman, D. 2004, A\&A 417, 751 (Erratum: A\&A 435, 339)

Auwers, A. 1891, Astron. Nachr. 128, 361

Böhm-Vitense, E. 1958, ZfA 46, 108

Bahcall, J. N. \& Pinsonneault, M. H. 1995, (with an appendix by G. J. Wasserburg), Rev. Mod. Phys. 67, 781

Bahcall, J. N. \& Serenelli, A. M. 2005, ApJ 626, 530

Bahcall, J. N., Gonzalez-Garcia, M. C., \& Peña-Garay, C. 2004, J. High Energy Phys. 08, 016

Bahcall, J. N., Basu, S., Pinsonneault, M., \& Serenelli, A. M. 2005, ApJ 618, 1049

Bahcall, J. N., Serenelli, A. M., \& Basu, S. 2006, ApJS 165, 400

Barnes, S. A. 2007, ApJ 669, 1167

Basu, S. \& Antia, H. M. 2008, Phys. Rep. 457, 217

Basu, S., Christensen-Dalsgaard, J., \& Thompson, M. J. 2002, in: F. Favata, I. W. Roxburgh \& D. Galadí-Enríquezi (eds), Proc. 1st Eddington Workshop, 'Stellar Structure and Habitable Planet Finding', ESA SP-485 (Noordwijk, The Netherlands: ESA), p. 249

Bonanno, A., Schlattl, H., \& Paternò, L. 2002, A\&A 390, 1115

Brown, T. M. \& Christensen-Dalsgaard, J. 1998, ApJ 500, L195

Chaplin, W. J., Elsworth, Y., Isaak, G. R., Miller, B. A., \& New, R. 2004, MNRAS 352, 1102

Chaplin, W. J., Serenelli, A. M., Basu, S., Elsworth, Y., New, R., \& Verner, G. A. 2007, ApJ 670,872

Chaplin, W. J., Houdek, G., Appourchaux, T., Elsworth, Y., New, R., \& Toutain, T. 2008a, $A \& \mathrm{~A} 485,813$

Chaplin, W. J., Appourchaux, T., Arentoft, T., et al. 2008b, Astron. Nach. 329, 549

Chaplin, W. J., Appourchaux, T., Arentoft, T., et al. 2008c, in: L. Gizon \& M. Roth (eds), Proc. HELAS II International Conference: Helioseismology, Asteroseismology and the MHD Connections, J. Phys.: Conf. Ser. 118, 012048

Christensen-Dalsgaard, J. 1984, in: A. Mangeney \& F. Praderie (eds), Space Research Prospects in Stellar Activity and Variability (Paris: Paris Observatory Press), p. 11

Christensen-Dalsgaard, J. 1988, in: J. Christensen-Dalsgaard \& S. Frandsen (eds), Advances in helio- and asteroseismology, Proc. IAU Symposium No 123 (Dordrecht: Reidel), p. 295

Christensen-Dalsgaard, J. 2002, Rev. Mod. Phys. 74, 1073

Christensen-Dalsgaard, J. \& Thompson, M. J. 1997, MNRAS 284, 527

Christensen-Dalsgaard, J., Däppen, W., \& Lebreton, Y. 1988, Nature 336, 634

Christensen-Dalsgaard, J., Däppen, W., Ajukov, S. V., et al. 1996, Science 272, 1286

Christensen-Dalsgaard, J., Arentoft, T., Brown, T. M., Gilliland, R. L., Kjeldsen, H., Borucki, W. J., \& Koch, D. 2007, in: G. Handler \& G. Houdek (eds), Proc. Vienna Workshop on the Future of Asteroseismology, Comm. in Asteroseismology 150, 350

Christensen-Dalsgaard, J., Di Mauro, M. P., Houdek, G., \& Pijpers, F. 2009, A\&A, in the press [arXiv:0811.1001 [astro-ph]].

Cunha, M. S. \& Metcalfe, T. S. 2007, ApJ 666, 413

Demarque, P., Guenther, D. B. \& Kim, Y.-C. 1999, ApJ 517, 510

Dziembowski, W. A. \& Goode, P. R. 1997, A\&A 317, 919 
Dziembowski, W. A., Fiorentini, G., Ricci, B., \& Sienkiewicz, R. 1999, A\&A 343, 990

Elsworth, Y., Howe, R., Isaak, G. R., McLeod, C. P., \& New, R. 1990, Nature 347, 536

Fröhlich, C. \& Lean, J. 2004, A\&AR 12, 273

Gough, D. O. 1987, Nature 326, 257

Gough, D. O. 1996, Observatory 116, 313

Gough, D. O. 2001, in: T. von Hippel, C. Simpson \& N. Manset (eds), Astrophysical Ages and Time Scales, ASP Conf. Ser. 245 (San Francisco: ASP), p. 31

Gough, D. O. 2002, in: F. Favata, I. W. Roxburgh \& D. Galadí-Enríquezi (eds), Proc. 1st Eddington Workshop: 'Stellar structure and habitable planet finding', ESA SP-485 (Noordwijk, The Netherlands: ESA), p. 65

Gough, D. O. \& Kosovichev, A. G. 1993, in: T. M. Brown (ed.), Proc. GONG 1992: Seismic investigation of the Sun and stars, ASP Conf. Ser. 42, (San Francisco: ASP), p. 351

Gough, D. O. \& Novotny, E. 1990, Solar Phys. 128, 143

Gough, D. O. \& Weiss, N. O. 1976, MNRAS 176, 589

Grevesse, N. \& Noels, A. 1993, in: N. Prantzos, E. Vangioni-Flam \& M. Cassé (eds), Origin and evolution of the Elements, (Cambridge: Cambridge Univ. Press), p. 15

Grundahl, F., Arentoft, T., Christensen-Dalsgaard, J., Frandsen, S., Kjeldsen, H., \& Rasmussen, P. K. 2008, in: L. Gizon \& M. Roth (eds), Proc. HELAS II International Conference: Helioseismology, Asteroseismology and the MHD Connections, J. Phys.: Conf. Ser. 118, 012041

Guenther, D. B. \& Demarque, P. 1997, ApJ 484, 937

Guzik, J. A. 2006, in: K. Fletcher (ed.), Proc. SOHO 18 / GONG 2006 / HELAS I Conf. Beyond the spherical Sun, ESA SP-624, (Noordwijk, The Netherlands: ESA).

Guzik, J. A. 2008, MemSAI 79, 481

Haxton, W. C. 2008, PASA 25, 44

Haxton, W. C., Parker, P. D., \& Rolfs, C. E. 2006, Nucl. Phys. A. 777, 226

Houdek, G. \& Gough, D. O. 2007a, MNRAS 375, 861

Houdek, G. \& Gough, D. O. 2007b, in: R. J. Stancliffe, J. Dewi, G. Houdek, R. G. Martin \& C. A Tout (eds), Unsolved Problems in Stellar Physics, AIP Conf. Proc. 948 (Melville: AIP), p. 219

Houdek, G. \& Gough, D. O. 2008, in: L. Deng \& K. L. Chan (eds), The Art of Modelling Stars in the 21st Century, Proc. IAU Symposium No 252 (Cambridge: Cambridge University Press), p. 149

Howe, R., Komm, R. W., \& Hill, F. 2002, ApJ 580, 1172

Iglesias, C. A. \& Rogers, F. J. 1996, ApJ 464, 943

Kjeldsen, H., Bedding, T. R., \& Christensen-Dalsgaard, J. 2008a, ApJ 683, L175

Kjeldsen, H., Bedding, T. R., \& Christensen-Dalsgaard, J. 2008b, in: F. Pont, D. Queloz \& D. D. Sasselov (eds), Transiting Planets, Proc. IAU Symposium No 253 (Cambridge: Cambridge University Press), in the press [arXiv:0807.0508v1 [astro-ph]]

Ludwig, H.-G., Freytag, B., \& Steffen, M. 1997, in: F. P. Pijpers, J. Christensen-Dalsgaard \& C. S. Rosenthal, C. S. (eds), SCORe'96: Solar Convection and Oscillations and their Relationship, (Dordrecht: Kluwer), p. 59

Ludwig, H.-G., Freytag, B., \& Steffen, M. 1999, A\&A 346, 111

Mazumdar, A., Basu, S., Collier, B. L., \& Demarque, P. 2006, MNRAS 372, 949

Monteiro, M. J. P. F. G., Christensen-Dalsgaard, J., \& Thompson, M. J. 2002, in: F. Favata, I. W. Roxburgh \& D. Galadí-Enríquezi (eds), Proc. 1st Eddington Workshop: 'Stellar structure and habitable planet finding', ESA SP-485 (Noordwijk, The Netherlands: ESA), p. 291

Mosser, B., Appourchaux, T., Catala, C., Buey, J.-T. and the SIAMOIS team 2008, in: L. Gizon \& M. Roth (eds), Proc. HELAS II International Conference: Helioseismology, Asteroseismology and the MHD Connections, J. Phys.: Conf. Ser. 118, 012042

Otí Floranes, H., Christensen-Dalsgaard, J., \& Thompson, M. J. 2005, MNRAS 356, 671

Robertson, R. G. H. 2006, Prog. Particle Nuclear Phys. 57, 90

Rogers, F. J. \& Nayfonov, A. 2002, ApJ 576, 1064

Rosenthal, C. S., Christensen-Dalsgaard, J., \& Nordlund, Å., Stein, R. F. \& Trampedach, R. 1999, A\&A 351, 689

Roxburgh, I. W. \& Vorontsov, S. V. 2003, A\&A 411, 215 
Roxburgh, I. W. 2002, in: F. Favata, I. W. Roxburgh \& D. Galadí-Enríquezi (eds), Proc. 1st Eddington Workshop: 'Stellar structure and habitable planet finding', ESA SP-485 (Noordwijk, The Netherlands: ESA), p. 75

Sackmann, I.-Juliana \& Boothroyd, A. I. 2003, ApJ 583, 1024

Thompson, M. J., Christensen-Dalsgaard, J., Miesch, M. S., \& Toomre, J. 2003, ARAA 41, 599

Toutain, T. \& Kosovichev, A. G. 2005, ApJ 622, 1314

Trampedach, R., Stein, R. F., Christensen-Dalsgaard, J., \& Nordlund, A\&A. 1999, in: A. Giménez, E.F. Guinan \& B. Montesinos (eds), Theory and Tests of Convection in Stellar Structure, ASP Conf. Ser. 173 (San Francisco: ASP), p. 233

Ulrich, R. K. 1986, ApJ 306, L37

\section{Discussion}

G. Meynet: Can you say a few words about the behavior of the angular velocity near the center of the Sun? Is it increasing or decreasing towards the center? What are the most recent results?

J. Christensen-Dalsgand: The data are consistent with constant rotation in the core. Unfortunately, improving the error bars with p-mode observations will require very extended observations and the g-mode claims, although very interesting, are so far tentative.

D. Soderblom: You mentioned that Kepler may be able to detect planets from phase shifts of oscillation frequencies. Has that effect been seen on the Sun?

J. Christensen-DalsgandD: I suppose that the effect of Jupiter might be visible, but it has not been seen, or looked for, as far as I know.

S. LegGett: Can you comment further on the Asplund abundances? Have they been revised upward?

J. Christensen-DalsgandD: There has been an independent analysis of a similar nature by Caffau et al. (2008, A \&A, 488, 1031); preliminary results show an oxygen abundance halfway between the old and the Asplund values.

P. Demarque: I draw your attention to a recent detailed review of the solar abundance problem by Basu \& Antia (2008, Phys. Rep., 457, 217). There is also a poster downstairs in which my collaborators and I point out some internal inconsistencies in the Asplund et al. analysis. Having worked on both helioseismology and 3-D simulations, I must say that I consider the seismic results to be more trustworthy. 


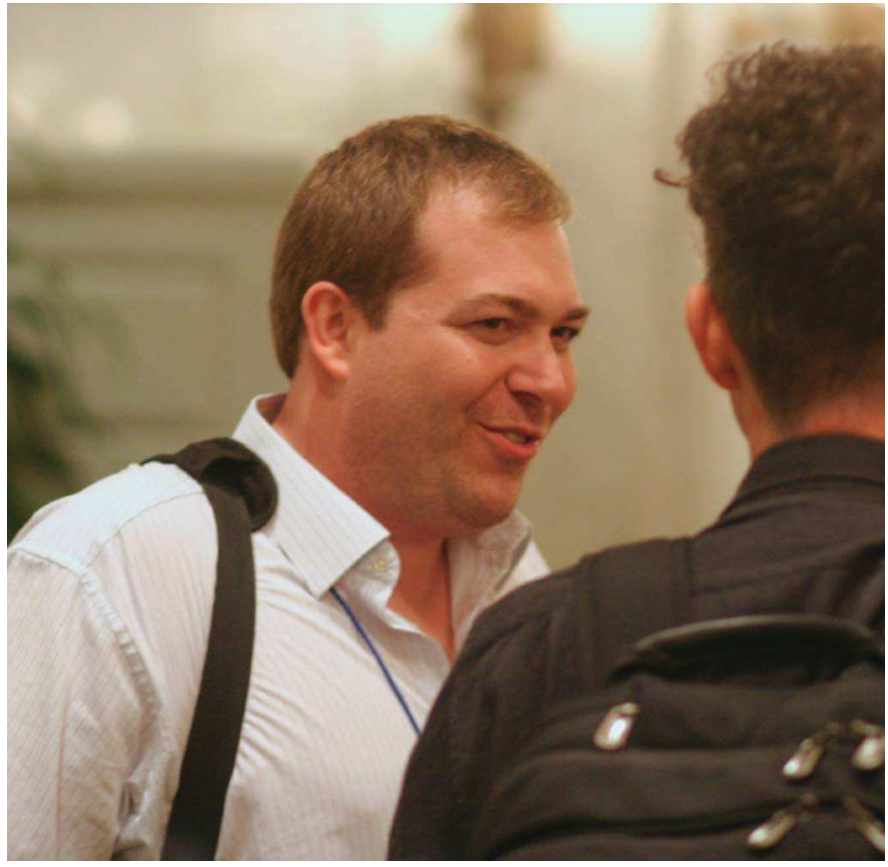

Eric Mamajek

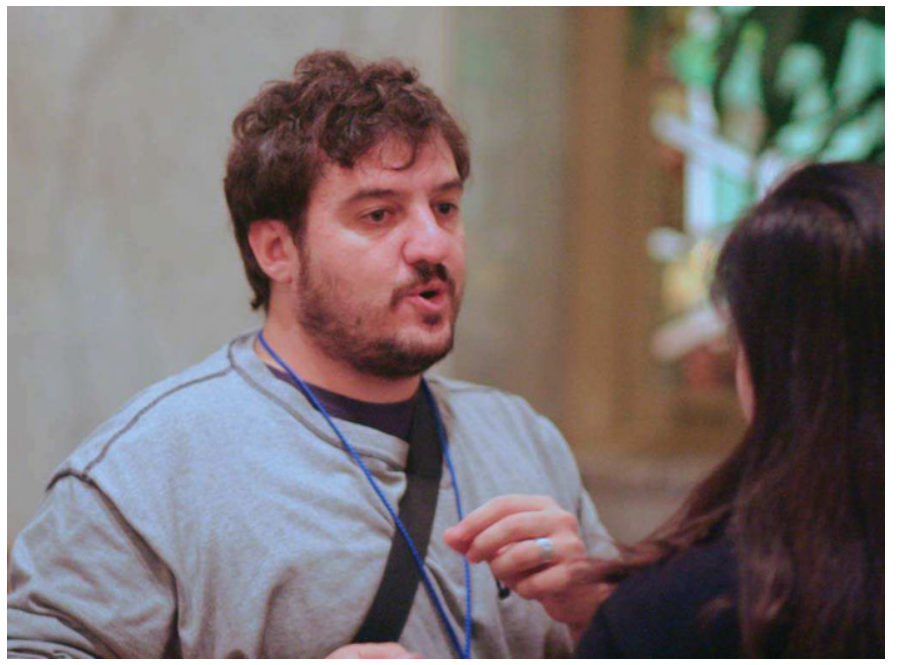

Matteo Monelli 